\title{
Effect of MDP-Based Primers on the Luting Agent Bond to Y-TZP Ceramic and to Dentin
}

\author{
Sheila Butler $\mathbb{D},{ }^{1}$ Bernie Linke, ${ }^{2}$ and Ysidora Torrealba $\mathbb{D}^{2}$ \\ ${ }^{1}$ School of Dentistry, Western University, London N6A 5C1, Canada \\ ${ }^{2}$ School of Dentistry, University of Alberta, Edmonton T6G 2R3, Canada \\ Correspondence should be addressed to Sheila Butler; sheila.butler@schulich.uwo.ca
}

Received 25 June 2018; Revised 3 August 2018; Accepted 2 September 2018; Published 16 September 2018

Academic Editor: Xiupeng Wang

Copyright (C) 2018 Sheila Butler et al. This is an open access article distributed under the Creative Commons Attribution License, which permits unrestricted use, distribution, and reproduction in any medium, provided the original work is properly cited.

Purpose. The aim of this study was to evaluate the influence of multimode MDP-based primers and different application protocols on the bond strength of a representative resin cement to an yttrium stabilized tetragonal zirconia (Y-TZP) ceramic. Materials and Methods. The occlusal dentin from 60 human molars was exposed. The teeth and zirconia cylinders $(\mathrm{N}=60)(3 \mathrm{~mm}$ of diameter; $4 \mathrm{~mm}$ of height) were divided into six groups $(\mathrm{n}=10)$ according to the ceramic surface conditioning: $(1)$ air abraded with $\mathrm{SiO}_{2}$ particles; (2) Z-Prime Plus; (3) air abraded with $\mathrm{SiO}_{2}$ particles + Z-Prime Plus; (4) air abraded with $\mathrm{SiO}_{2}$ particles + All-Bond Universal; (5) air abraded with $\mathrm{SiO}_{2}$ particles + ScotchBond Universal Adhesive; and (6) untreated zirconia. The luting agent (DuoLink cement) was applied on the treated dentin surface. Specimens were stored in water $\left(37^{\circ} \mathrm{C}, 24 \mathrm{~h}\right)$ and tested in shear bond strength. Data were statistically analyzed using 2-way ANOVA and Post hoc Tukey tests $(\alpha=0.05)$. Results. Significant effects of ceramic conditioning were found $(\mathrm{p}<0.0001)$. The specimens sandblasted with silica particles followed by the application of Z-Prime Plus or All-Bond Universal presented greater bond strength values. For the untreated zirconia, several specimens failed prematurely prior to testing. Conclusions. Sandblasting with silica particles combined with Z-Prime Plus increased the bond strength.

\section{Introduction}

Yttrium stabilized tetragonal zirconia polycrystals (Y-TZP) ceramic is a material used for the restoration of teeth with primarily full coverage restorations. The zirconia crowns can be milled monolithically to full contour or alternatively the core of zirconia can be milled followed by layering of feldspathic porcelain onto the core. The layering of the porcelain onto the core of the zirconia has resulted in superior esthetics along with issues of chipping of the veneered porcelain [1-3].

In some situations, the remaining tooth structure for a full coverage crown does not allow for adequate retention/resistance form. Therefore, the options are either increasing the clinical crown height with crown-lengthening procedures to facilitate restorative therapy $[4,5]$ or increasing the bond of the cement to the remaining tooth structure [6] and the zirconia [7].

Zirconia does not contain an amorphous silica glass and as a result does not allow for traditional ceramic surface treatment $[8,9]$. Various surface treatments, both mechanical and chemical, of zirconia have been attempted with varying results. These treatments include ultrasonic cleaning, abrasion, sandblasting, hydrofluoric acid etching, silanization, and application of surface bonding adhesives [10, 11].

One of the ongoing concerns with increasing the internal surface area for bonding by mechanically altering the surface of zirconia with a diamond bur or sandblasting is the development of a phase change in the zirconia and as a result introducing flaws into zirconia microstructure [12-14]. The resulting reduction in physical properties of the zirconia could result in a weaker restoration and compromising the long-term prognosis. Recent research would indicate that this may not be the case and under certain sandblasting conditions may actually increase the zirconia strength due to transformational toughening, along with providing some surface roughness for micromechanical retention possibilities for cement of zirconia $[5,14,15]$.

The use of MDP (10-methacryloyloxydecyl dihydrogen phosphate) as a multimode functional adhesive monomer has been postulated to provide increased retention for cements 
used in the cementing of zirconia crowns by increasing the bond to zirconia as well as to dentin [10, 11]. It has been proposed that the methacryloxy end of the phosphate compound couples with the resin matrix, and phosphoric acid group react with the zirconia surface. The ability of surface treatments to improve the retention of cements to zirconia has had varying results.

The aim of this study is to evaluate the influence of various multimode MDP-based primers and different application protocols on the bond strength of a representative resin cement to a yttrium stabilized tetragonal zirconia (Y-TZP) ceramic as well as of zirconia to dentin.

\section{Materials and Methods}

Partially sintered zirconia disks (NexxZr, Sagemax Bioceramic, Berlin, Germany, Shade: WT, LOT: HFMCG) were used to obtain zirconia cylinders of $4 \mathrm{~mm}$ in height and 3 $\mathrm{mm}$ in diameter using a computer-aided manufacturing technique (vhf CAM S1 Impression Milling Machine, Ammerbuch, Germany). Sixty zirconia cylinders were machined under water coolant (IsoMet ${ }^{\circledR}$ 1000, Buehler, Lake Bluff, IL, USA). The cylinders were subsequently sintered in a VITA Zyrcomat 6000 MP furnace (VITA, Bad Säckingen, Germany), according to the manufacturer's instructions. All specimens were polished using Diamond Lapping Films (Allied High Tech Products Inc, Compton, CA, USA) in a decreasing sequence of abrasive size from $30,15,9$ to $3 \mu \mathrm{m}$, under copious water cooling.

Sixty noncarious human molars extracted for periodontal or orthodontic reasons were collected under ethical approval from the University of Alberta Ethics Committee In Human Research (Pro00063336). The teeth were scaled, cleaned, stored in $0.5 \%$ chloramine solution at $4^{\circ} \mathrm{C}$ to prevent bacterial growth, and used within 3 months following extraction. The crowns were sectioned horizontally with a low speed and intense water cooling to remove the occlusal morphology. The teeth were then ground perpendicularly to their longitudinal axes using P320 SiC paper (Schmitz Metallographie $\mathrm{GmbH}$, Herzogenrath, Germany) until all occlusal enamel was completely removed $[16,17]$. The teeth were embedded in acrylic resin (Orthodontic Resin, Dentsply) up to $2.0 \mathrm{~mm}$ below the cemento-enamel junction. The ground dentin surface was then cleaned using a rubber cup and fine pumice water slurry. Subsequently a smear layer was created by wet-grinding the dentin surface with $\mathrm{P} 600 \mathrm{SiC}$ paper for $60 \mathrm{~s}$. The prepared coronal dentin was treated as follows: lightly air-dried in an oil free air-stream and then etched with $37 \%$ phosphoric acid for $15 \mathrm{~s}$ and washed with $10 \mathrm{~mL}$ of distilled water and the excess water was removed with absorbent paper. Two layers of adhesive (All-Bond Universal, Bisco, Schaumburg, IL, USA, LOT: 1600004095) were applied to the coronal dentin. For each layer any excess adhesive was removed with a dry microbrush, prior to gentle air-drying. The adhesive was light cured for $10 \mathrm{~s}$ perpendicular to the dentine surface from a distance of $2 \mathrm{~mm}$ using a Mini LED (Satelec Acteon) with an irradiance of $1250 \mathrm{~mW} / \mathrm{cm}^{2}$. The prepared teeth were finally randomly assigned to six groups of ten teeth.
Prior to surface conditioning, all ceramic blocks were ultrasonically cleaned (Bransonic ${ }^{\circledR}$ ultrasonic cleaner, Branson Ultrasonic, Danbury, CT, USA) for 5 minutes in distilled water. The ceramic cylinders were randomly divided into six groups $(n=10)$ with each group receiving a different conditioning treatment to the bonding surface. Group (1) was air abraded with $\mathrm{SiO}_{2}$ particles; (2) primed with a thin layer of Z-Prime Plus (Bisco, Schaumburg, IL, USA, LOT: 1600004092); (3) air abraded with $\mathrm{SiO}_{2}$ particles and primed with a thin layer of Z-Prime Plus; (4) air abraded with $\mathrm{SiO}_{2}$ particles and coated with a thin layer of All-Bond Universal; (5) air abraded with $\mathrm{SiO}_{2}$ particles and coated with a thin film of ScotchBond Universal Adhesive (3M ESPE, Seefeld, Germany, LOT: 632027). A final group of ten specimens (6) were left as the untreated zirconia control.

Particle air abrasion was performed by making circular movements with the nozzle of the air abrasion unit at a distance of $10 \mathrm{~mm}$, with 2.8-bar pressure for $15 \mathrm{~s}$ using $30 \mu \mathrm{m}$ diameter $\mathrm{SiO}_{2}$ particles (Rocatec ${ }^{\mathrm{TM}}$ Soft, 3M ESPE, Seefeld, Germany, LOT: 450384).

A small portion of Duo-link cement (Bisco, Schaumburg, IL, USA, LOT: 1600003948) was placed on the treated ceramic surface. The zirconia cylinder was placed on the treated dentin surface using a positioning device that allowed for the application of a consistent $750 \mathrm{~g}$ load on the ceramic-resin cement-dentin block. Excess cement was carefully removed using a microbrush and the resin cement light cured (Mini LED Satelec Acteon, $1250 \mathrm{~mW} / \mathrm{cm}^{2}$ ) for $10 \mathrm{~s}$ each from two opposite sides of the ceramic cylinder. After ten minutes following resin cementation, the specimens were transferred to storage in distilled water for $24 \mathrm{~h}$ at $37^{\circ} \mathrm{C}$ prior to shear bond strength determination.

Shear bond testing was performed in a universal testing machine (ElectroPuls 3000, Instron GmbH, Darmstadt, Germany) with loading applied in a knife-edge configuration using a crosshead speed of $0.5 \mathrm{~mm} / \mathrm{min}$. Each specimen was rigidly positioned to ensure that the adhesive interface was parallel and as close as possible to the knife displacement axis. The shear bond strength (SBS) $(\mathrm{MPa})$ was calculated from the recorded failure load $(P)$ and the cross-sectional area of the adhesive interface where

$$
S B S=\frac{P}{\pi r^{2}}
$$

and $r$, the radius of the bonding interface, was $1.5 \mathrm{~mm}$.

The fractured surfaces of all the tested specimens were visualized in a stereo microscope at up to 50x magnification (Leica M125, Wetzlar, Germany) to discriminate the failure mode. Further specimens with representative fractures were selected for imaging using scanning electron microscopy (SEM). Specimens were mounted on a metallic stub, sputter coated with $7 \mathrm{~nm}$ of platinum in a Polaron E5100 coating unit (Polaron Equipment, Ltd., Bedford, UK), and observed at x20 and x500 magnifications (Hitachi S-2500, Hitachi, Mito City, Japan).

The shear bond strength $(\mathrm{MPa})$ data were statistically analyzed using a two-way analysis of variance (ANOVA) where the factors were surface finish (at two levels) and the use of a surface primer/adhesive (at four levels) followed by 
TABLE 1: Two-way ANOVA results of the shear bond strength (SBS) data.

\begin{tabular}{lccccc}
\hline Source & DF & SS & MS & F value $^{*}$ & $\boldsymbol{p ~ v a l u e}^{*}$ \\
\hline Corrected Model & 5 & 3829 & 766 & 17.2 & 0.001 \\
Factor 1: Surface Finish & 1 & 1043 & 1043 & 442 & 0.001 \\
Factor 2: Conditioning Primer & 3 & 1327 & 1157 & 9.9 & 0.001 \\
Factorial Interaction & 1 & 1157 & & 25.9 & 0.001 \\
Corrected Total & 59 & 6240 & & \\
\hline
\end{tabular}

${ }^{*} \mathrm{p}<0.05$.

TABLE 2: Mean values (MPa) and standard deviations (in parentheses) of the shear bond strength obtained for the different ceramic surface conditioning.

\begin{tabular}{lccccc}
\hline $\begin{array}{l}\text { Untreated } \\
\text { zirconia }\end{array}$ & SB only & $\begin{array}{c}\text { Z-Prime Plus } \\
\text { only }\end{array}$ & SB + Z-Prime Plus & $\begin{array}{c}\text { SB + All-Bond } \\
\text { Universal }\end{array}$ & $\begin{array}{c}\text { SB + ScotchBond } \\
\text { Universal } \\
\text { Adhesive }\end{array}$ \\
\hline 5.45 & 4.91 & 5.18 & 26.15 & 18.56 & 11.83 \\
$(6.34)^{\mathrm{a}}$ & $(1.07)^{\mathrm{a}}$ & $(4.11)^{\mathrm{a}}$ & $(9.41)^{\mathrm{b}}$ & $(9.42)^{\mathrm{b}, \mathrm{c}}$ & $(5.69)^{\mathrm{a}, \mathrm{c}}$ \\
\hline
\end{tabular}

Identical letters indicate no statistically significant differences $(P>.05)$.

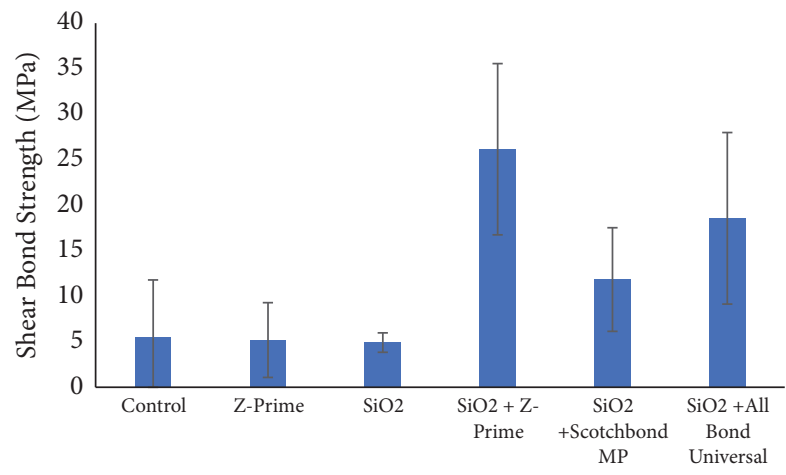

Figure 1: The mean bond strength values (MPa) according to the surface treatment.

post hoc Tukey tests $(\alpha=0.05)$. P values less than 0.05 were considered to be statistically significant in all tests. Specimens that failed prematurely during the aging conditions were considered as $0 \mathrm{MPa}$ for statistical analysis.

\section{Results}

A two-way ANOVA demonstrated a significant impact of modifying the zirconia surface with $\mathrm{SiO}_{2}$ particle air abrasion prior to cementation on the recorded shear bond strength (SBS) $(\mathrm{p}<0.001)$. Furthermore, the SBS was significantly influenced by the use of a surface primer/adhesive but the pattern of change in SBS was associated dependent on zirconia surface condition ( $\mathrm{p}<0.001)$. Post hoc Tukey tests demonstrated that the use of Z-Prime Plus and All-Bond Universal to condition the $\mathrm{SiO}_{2}$ particle air abraded zirconia surface, resulted in significantly higher bond strengths $(\mathrm{p}<0.001)$ than the corresponding unconditioned surface (Table 1). The mean bond strength values and associated standard deviations for each group are shown in Table 2 and Figure 1. No surface modification, $\mathrm{SiO}_{2}$ particle air abrasion only, and use of Z-Prime Plus only promoted low and unstable bond strengths with several pretest failures recorded. In contrast, air abrasion with silica particles resulted in higher and more consistent bond strengths (less pretest failures) when combined with Z-Prime Plus and also All-Bond Universal. Air abrasion with silica particles in combination with ZPrime Plus or All-Bond Universal allowed the highest mean SBS values.

A summary of failure types is presented in Table 3 and representative micrographs of the debonded surfaces are exhibited in Figure 1. Adhesive failures (between the luting cement and Y-TZP surface) took place for the majority of specimens. No adhesive failures involving the cementdentin surface were observed. In addition, mixed failures were observed as shown in Figure 2.

\section{Discussion}

This study evaluated the influence on bond strength of multimode MDP-based primers and application protocols on the bond strength of resin cement to dentin and a yttrium stabilized tetragonal zirconia polycrystal (Y-TZP) ceramic.

The shear bond strength test (SBS) was used to evaluate the adhesive interface between dentin, resin cement, surface treatment modalities, and the Y-TZP. Shear bond strength tests evaluate the cohesive strength of the base material better than the bond strength of the adhesive interface [18]. The concern with a SBS is that the test increasingly stresses the base components with possible deformation of those substrates and this flexing may actually test the resistance of the components to bending forces of the substrates and not the adhesive complex. The examination of the fractured surfaces in this current study exhibited the predominance of adhesive failures between ceramic and cement as shown in Table 3 .

There is a concern with air abrasion to Y-TZP because this process may induce stresses that result in crack propagation [19-21]. Recently a study using 4-point flexural test indicated 
TABLE 3: Number of specimens per group (n), number of pretest failures (PTF), and failure types of the debonded specimens. Failure between ceramic and cement (Adhes-C-cem); failure between dentin and cement (Adhes-D-cem); cohesive failure of cement and ceramic (MIX); cohesive failure of the ceramic (C-cer); cohesive failure of the cement (C-cem).

\begin{tabular}{|c|c|c|c|c|c|c|c|}
\hline \multirow{2}{*}{$\begin{array}{l}\text { Study factors } \\
\text { Ceramic conditioning }\end{array}$} & \multirow{2}{*}{$\mathbf{n}$} & \multirow{2}{*}{ PTF } & \multicolumn{5}{|c|}{ FAILURE TYPES } \\
\hline & & & Adhes-C-cem & Adhes-D-cem & C-cer & C-cem & MIX \\
\hline Untreated zirconia & 10 & 4 & 10 & 0 & 0 & 0 & 0 \\
\hline SB only & 10 & 0 & 6 & 0 & 0 & 3 & 1 \\
\hline Z-Prime Plus only & 10 & 1 & 7 & 0 & 0 & 0 & 3 \\
\hline SB + Z-Prime Plus & 10 & 0 & 8 & 0 & 0 & 2 & 0 \\
\hline SB + All-Bond Universal & 10 & 0 & 3 & 0 & 0 & 1 & 6 \\
\hline SB + ScotchBond Universal Adhesive & 10 & 0 & 5 & 0 & 0 & 3 & 2 \\
\hline
\end{tabular}

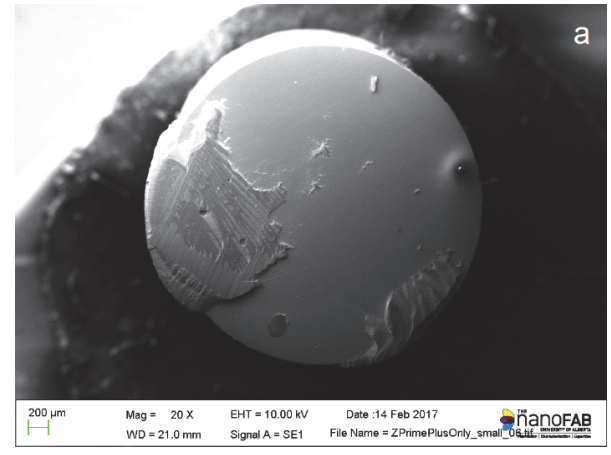

(a)

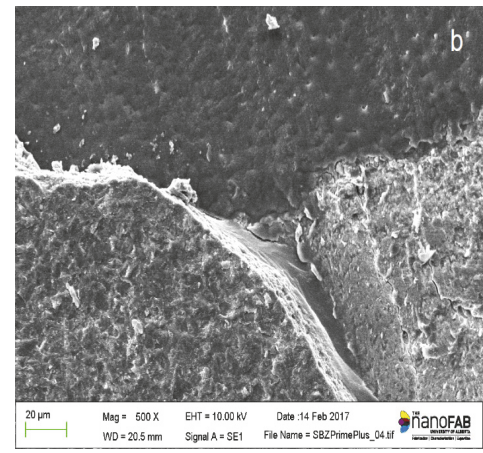

(b)

Figure 2: Representative micrographs of the debonded surfaces: (a) mixed failure - residue of cement left on the left side of the debonded surface from Z-Prime Plus only group; (b) mixed failure exhibiting dentin tubules and cement from SB + Z-Prime Plus group.

that if 30 micron Si-coated Aluminum oxide was used at 10 mm with 2.8-bar pressure for 15 seconds then there would be no reduction in flexural strength. In this study, 30 micron $\mathrm{SiO}_{2}$ was used [14].

Significant effects of ceramic conditioning were found $(\mathrm{p}<0.0001)$. The specimens air abraded with $\mathrm{SiO}_{2}$ particles followed by the application of Z-Prime Plus or All-Bond Universal resulted in higher bond strength values. For the untreated zirconia, several specimens failed prematurely prior to testing which is attributed to the unstable and weak bond strength present in this group.

The results of this study would indicate that air abrasion with $30 \mu \mathrm{m}$ of $\mathrm{SiO}_{2}$ particle with no further surface treatment does not improve the bond strength. However, if subsequent to air abrasion a multimode MDP application is completed to Y-TZP, there is a significant difference in the bond strength. There have been various explanations provided for this fact, including (a) mechanical surface abrasion and cleaning of the surface, (b) silica deposition, (c) increased chemical reactivity energy levels, or (d) a combination of all the preceding reasons [22].

There are differences among the studies regarding sandblasting consequences likely due to the varying size of particles, pressure, distance, and angle of application. Nevertheless, surface roughness alone would not account for improved bond strength as there was no significant difference in bond strength between untreated zirconia and the specimens which were only sandblasted. Additionally, this study demonstrated that without air abrasion the application of a multimode MDP adhesive monomer did not provide an increase in bonding strength.

Chen in 2011 [22] has shown that sandblasting with Sicoated alumina particles results in a surface layer of $\mathrm{SiO}_{2}$ deposited on the zirconia surface resulting in increased reactivity between that layer and a MDP adhesive monomer such as All-Bond Universal and ScotchBond Universal. The phosphate ester monomers from MDP have been shown to react with Y-TZP [23].

Z-Prime is a zirconia primer that contains a mixture of organophosphate and carboxylate monomers. The organophoshate monomer has an organofunctional end that can polymerize with the resin cement [13]. The synergistic coupling of phosphate and carboxyl functional monomers can bond to the metal oxides with the Y-TZP resulting in an increase in the bond. The findings of this study would confirm that observations as the highest values were obtained by SB + Z-Prime Plus. There was no significant difference between SB + Z-Prime Plus and SB + All-Bond Universal. All-Bond Universal and ScotchBond Universal are a multimode MDP primer. Although the bonding values were higher for $\mathrm{SB}+$ ScotchBond Universal than untreated zirconia, there was no significant difference between these two groups. This could be a result of the slightly lower $\mathrm{pH}$ value provided by the ScotchBond Universal resulting in decreased copolymerization of the resin cement used in this study. 
Evaluation of the mode of failure indicated that no complete failures occurred at the dentin- cement interface and this is consistent with the findings of other studies [24]. This indicated the bond to dentin is greater than the bond to zirconia.

Failures for SB + Z-Prime Plus took place mainly at the YTZP and cement interface. Given that all samples failed in the untreated zirconia at this interface this is not an unexpected finding and would indicate that given zirconia's dense tetragonal structure there is minimal mechanical retention provided by the material itself. When the zirconia was sandblasted there were less failures at the cement-zirconia interface indicating some change has occurred to the Y-TZP surface. The addition of Z-Prime Plus to the untreated surface changed the mode of failure in some samples but did not increase the bond strength for that of the untreated zirconia. When the samples were sandblasted and then conditioned with ZPrime Plus, All-Bond Universal, or ScotchBond Universal, changes occurred to the mode of failure compared to the untreated zirconia. This factor indicates a positive effect on bond strength at the Y-TZP-cement interface.

\section{Conclusion}

Within the limitations of this study, Y-TZP surface sandblasted with 30 micron $\mathrm{SiO}_{2}$ particles prior to conditioning the bonding surface significantly increased the bonding of YTZP to dentin. The SB + Z-Prime Plus protocol resulted in the highest bonding values.

\section{Clinical Relevance}

Zirconia surface air abraded with $\mathrm{SiO}_{2}$ particles and primed with a thin layer of Z-Prime Plus is the recommended surface treatment before cementing a zirconia-based restoration.

\section{Data Availability}

The data used to support the findings of this study are available from the corresponding author upon request.

\section{Conflicts of Interest}

The authors declare that they have no conflicts of interest.

\section{References}

[1] R. P. Christensen and B. J. Ploeger, "A clinical comparison of zirconia, metal and alumina fixed-prosthesis frameworks veneered with layered or pressed ceramic: a three-year report," The Journal of the American Dental Association, vol. 141, no. 11, pp. 1317-1329, 2010.

[2] A. D. Bona and J. R. Kelly, "The Clinical Success Of All-Ceramic Restorations," The Journal of the American Dental Association, vol. 139, pp. S8-S13, 2008.

[3] S. P. Passos, L. G. May, D. C. Barca, M. Özcan, M. A. Bottino, and L. F. Valandro, "Adhesive quality of self-adhesive and conventional adhesive resin cement to Y-TZP ceramic before and after aging conditions," Operative Dentistry, vol. 35 , no. 6, pp. 689-696, 2010.

[4] T. J. Hempton and J. T. Dominici, "Contemporary CrownLengthening Therapy," The Journal of the American Dental Association, vol. 141, no. 6, pp. 647-655, 2010.

[5] I. Pilalas, L. Tsalikis, and D. N. Tatakis, "Pre-restorative crown lengthening surgery outcomes: a systematic review," Journal of Clinical Periodontology, vol. 43, no. 12, pp. 1094-1108, 2016.

[6] A. Afrasiabi, E. Mostajir, and N. Golbari, "The effect of Z-primer on the shear bond strength of zirconia ceramic to dentin: in vitro," Journal of Clinical and Experimental Dentistry, vol. 10, pp. e661-e664, 2018.

[7] P. Thammajaruk, M. Inokoshi, S. Chong, and M. Guazzato, "Bonding of composite cements to zirconia: A systematic review and meta-analysis of in vitro studies," Journal of the Mechanical Behavior of Biomedical Materials, vol. 80, pp. 258-268, 2018.

[8] S. S. Atsu, M. A. Kilicarslan, H. C. Kucukesmen, and P. S. Aka, "Effect of zirconium-oxide ceramic surface treatments on the bond strength to adhesive resin," Journal of Prosthetic Dentistry, vol. 95, no. 6, pp. 430-436, 2006.

[9] M. A. Bottino, L. F. Valandro, R. Scotti, and L. Buso, "Effect of surface treatments on the resin bond to zirconium-based ceramic," International Journal of Prosthodontics, vol. 18, no. 1, pp. 60-65, 2005.

[10] J. P. Matinlinna, T. Heikkinen, M. Özcan, L. V. J. Lassila, and P. K. Vallittu, "Evaluation of resin adhesion to zirconia ceramic using some organosilanes," Dental Materials, vol. 22, no. 9, pp. 824-831, 2006.

[11] S. P. Passos, B. Linke, P. W. Major, and J. A. Nychka, "The effect of air-abrasion and heat treatment on the fracture behavior of Y-TZP," Dental Materials, vol. 31, no. 9, article no. 2568, pp. 10111021, 2015.

[12] S. Bhargava, H. Doi, and R. Kondo, "Effect of sandblasting on the mechanical properties of Y-TZP zirconia," Bio-Medical Materials and Engineering, vol. 22, pp. 383-398, 2012.

[13] L. G. May, S. P. Passos, D. B. Capelli, M. Özcan, M. A. Bottino, and L. F. Valandro, "Effect of silica coating combined to a MDPbased primer on the resin bond to Y-TZP ceramic," Journal of Biomedical Materials Research Part B: Applied Biomaterials, vol. 95, no. 1, pp. 69-74, 2010.

[14] M. Özcan, R. M. Melo, R. O. A. Souza, J. P. B. Machado, L. Felipe Valandro, and M. A. Botttino, "Effect of air-particle abrasion protocols on the biaxial flexural strength, surface characteristics and phase transformation of zirconia after cyclic loading," Journal of the Mechanical Behavior of Biomedical Materials, vol. 20, pp. 19-28, 2013.

[15] H. Sato, K. Yamada, G. Pezzotti, M. Nawa, and S. Ban, "Mechanical properties of dental zirconia ceramics changed with sandblasting and heat treatment," Dental Materials, vol. 27, no. 3, pp. 408-414, 2008.

[16] L. Cheng, K. Zhang, M. A. S. Melo, M. D. Weir, X. Zhou, and H. H. K. Xu, "Anti-biofilm dentin primer with quaternary ammonium and silver nanoparticles," Journal of Dental Research, vol. 91, no. 6, pp. 598-604, 2012.

[17] Y. Zhang, B. R. Lawn, E. D. Rekow, and V. P. Thompson, "Effect of sandblasting on the long-term performance of dental ceramics," Journal of Biomedical Materials Research Part B: Applied Biomaterials, vol. 71, no. 2, pp. 381-386, 2004.

[18] A. Della Bona and R. Van Noort, "Shear vs. Tensile Bond Strength of Resin Composite Bonded to Ceramic," Journal of Dental Research, vol. 74, no. 9, pp. 1591-1596, 1995. 
[19] I. Çağlar and N. Yanlkoğlu, "The Effect of Sandblasting, Er:YAG Laser, and Heat Treatment on the Mechanical Properties of Different Zirconia Cores," Photomedicine and Laser Surgery, vol. 34, no. 1, pp. 17-26, 2016.

[20] I. Sailer, A. Fehér, F. Filser, L. J. Gauckler, H. Lüthy, and C. H. F. Hämmerle, "Five-year clinical results of zirconia frameworks for posterior fixed partial dentures," International Journal of Prosthodontics, vol. 20, no. 4, pp. 383-388, 2007.

[21] S. S. Scherrer, M. Cattani-Lorente, E. Vittecoq, F. De Mestral, J. A. Griggs, and H. W. A. Wiskott, "Fatigue behavior in water of Y-TZP zirconia ceramics after abrasion with $30 \mu \mathrm{m}$ silica-coated alumina particles," Dental Materials, vol. 27, no. 2, pp. e28-e42, 2011.

[22] L. Chen, B. I. Suh, J. Kim, and F. R. Tay, "Evaluation of silicacoating techniques for Zirconia bonding," American Journal of Dentistry, vol. 24, pp. 79-84, 2011.

[23] L. Chen, B. D. Hammond, G. Alex, and B. I. Suh, "Effect of silane contamination on dentin bond strength," Journal of Prosthetic Dentistry, vol. 117, no. 3, pp. 438-443, 2017.

[24] Jin-Soo Ahn, Young-Ah Yi, Yoon Lee, and Deog-Gyu Seo, "Shear Bond Strength of MDP-Containing Self-Adhesive Resin Cement and Y-TZP Ceramics: Effect of Phosphate MonomerContaining Primers," BioMed Research International, vol. 2015, Article ID 389234, 6 pages, 2015. 


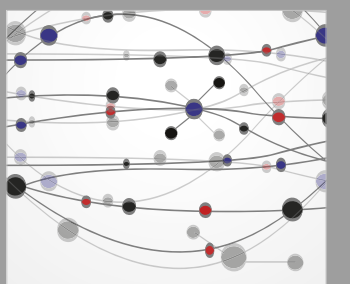

The Scientific World Journal
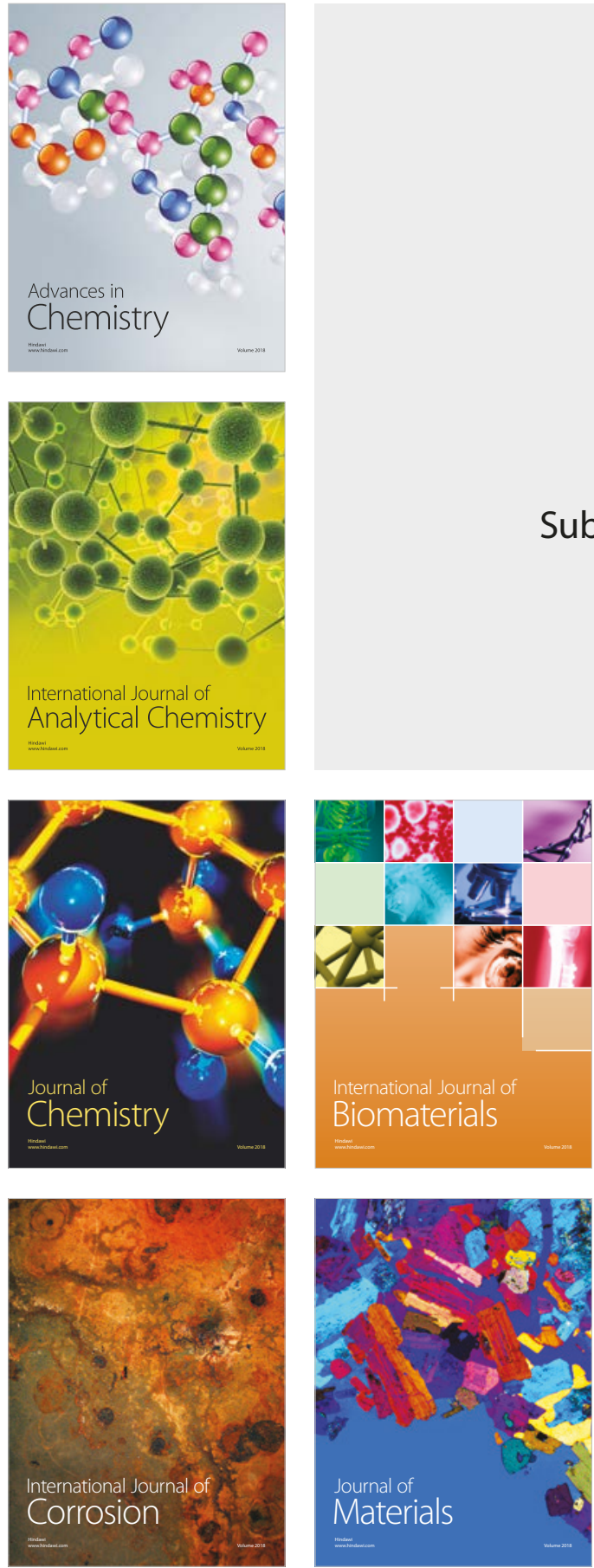

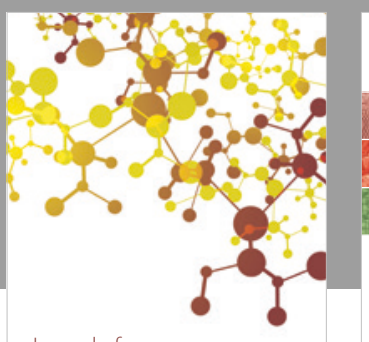

Journal of

Applied Chemistry
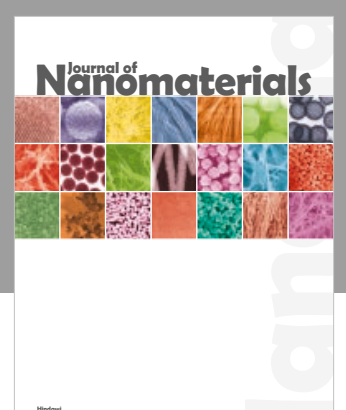

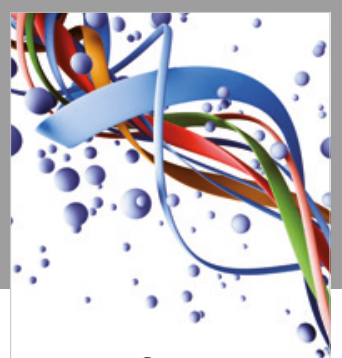

Scientifica

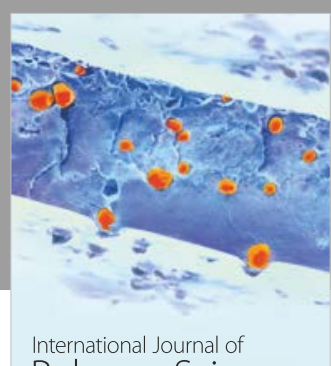

Polymer Science

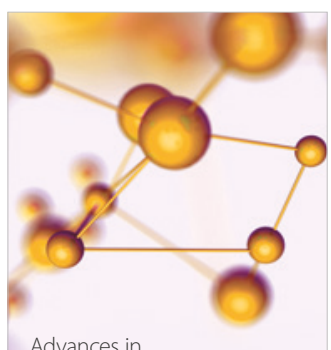

Physical Chemistry
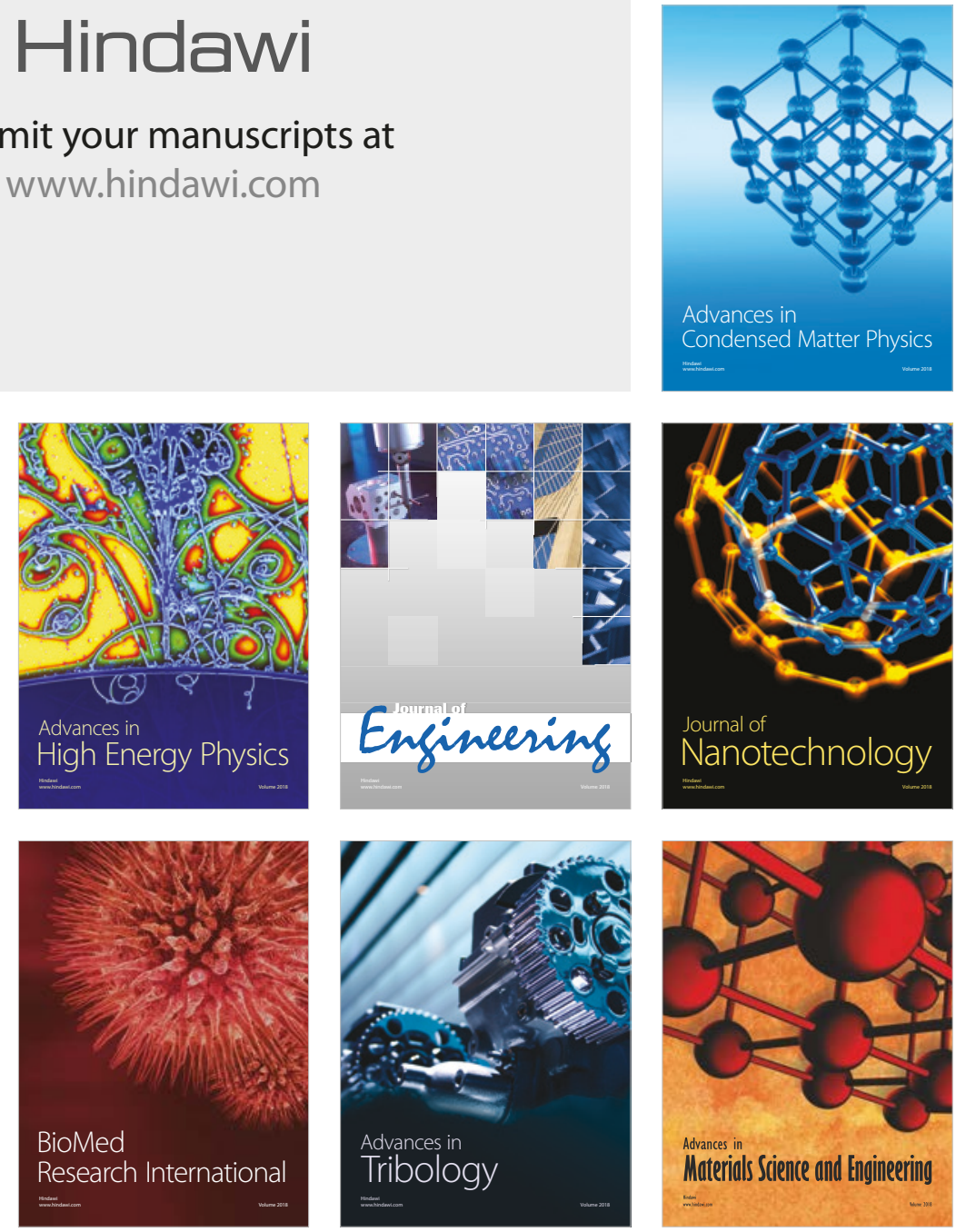